\title{
IV. RESULTS OF SHORE LABORATORY STUDIES ON MESOZOIC PLANKTONIC FORAMINIFERA FROM LEG 26 SITES 255, 256, 257, AND 258
}

\author{
Emile A. Pessagno, Jr., Geosciences Division, University of Texas at Dallas, Dallas, Texas \\ and \\ Fouad Y. Michael, Atlantic Richfield Company, Geological Science Group, Dallas, Texas
}

The zones and subzones used in this report are those proposed by Pessagno, $(1967,1969)$. Some samples contained Radiolaria; though poorly preserved, they were identified whenever possible. The radiolarian classification and zonation given by Pessagno (in press) is herein followed. Due to the sparsity of the fauna, the data was not tabulated.

$\mathrm{TA}=$ Total abundance of all planktonic foraminifera in the sample.

TA rads $=$ Total abundance of all Radiolaria in the sample.

RA = Relative abundance of each taxon. Symbols used are:

0 -Absent

1-Rare (1-10 specimens)

2-Moderately rare (11-25 specimens)

3-Common (26-50 specimens)

4-Abundant (51-100 specimens)

5 -Very abundant ( $>100$ specimens)

\section{SITE 255}

26-255-10-1, 21-24 cm.

Hard limestone and chert, no foraminifera were isolated. Inoceramus prisms are frequent. Pyritized spherical and discoidal pellets are common, coprolites?

Biostratigraphic determination: Cretaceous?

\section{SITE 256}

26-256-6-6, 105-109 cm. TA: 0. TA rads: 3 .

\section{RA}

2 Alievium gallowayi (White)

3 Dictyomitra formosa Squinabol

3 Dictyomitra napaensis Pessagno

2 Dictyomitra torquata? Forman

3 Praeconocaryomma universa Pessagno

3 Pseudoaulophacus floresensis Pessagno

3 Pseudoaulophacus lenticulatus (White)

Biostratigraphic determination: Alievium gallowayi Zone to Crucella espartoensis Zone, Patulibrachium lawsoni Subzone. Early Santonian to middle Campanian.

26-256-7-3,95-97 cm. TA: 0. TA rads: 3 .

RA

1 Dictyomitra pseudomacrocephala Squinabol (reworked?)

1 Dictyomitra sp.

$4 \quad$ Praeconocaryomma universa Pessagno

1 Pseudoaulophacus floresensis Pessagno
Biostratigraphic determination: The Dictyomitra sp. found in this sample is similar to a form reported in the Albian of California by Pessagno. The D. pseudomacrocephala is an Aptian-Cenomanian form; it is probably reworked. The same early Santonian to middle Campanian age is assigned to this core.
26-256-8-1, 60-63 cm.
TA: 0 .
26-256-8-3, 75-77 cm.
TA: 0 .
26-256-8-6, 75-79 cm.
TA: 0 .

In these three samples, few broken benthonic forms were reported.

26-256-9-1, 127-131 cm.

TA: 2 .

\section{RA \\ 1 Hedbergella planispira (Tappan) \\ 1 Hedbergella sp.}

Biostratigraphic determination: Probably Albian to Cenomanian?

\section{SITE 257}

26-257-4-2, 75-79 cm.

TA: 0

Sponge spicules abundant.

26-257-5-2, 75-79 cm.

TA: 0 .

26-257-6-1, 60-69 cm.

TA: 0 .

26-257-7-2, 75-79 cm.

TA: 0 .

26-257-7-5, 70-75 cm.

TA: 0 .

Sparse, badly preserved benthonic forms were reported in these samples.

Biostratigraphic determination: Undetermined.

26-257-8-1, 99-104 cm.

TA: 1 .

\section{RA}

1 Hedbergella planispira (Tappan)

1 Hedbergella amabilis Loeblich and Tappan Benthonic forms common.

26-257-8-2, 100-105 cm.

TA: 1 .

RA

1 Hedbergella planispira (Tappan)

1 Hedbergella sp.

Benthonic forms abundant.

Biostratigraphic determination: These two samples of Core 8 are probably of Albian or Cenomanian age. 
26-257-9-2, 29-31 cm.

TA: 0.

Benthonic forms abundant.

Biostratigraphic determination: Probably middle Albian via superposition.

26-257-10-1, 101-103 cm. TA: 0 . TA rads: 5 .

\section{RA}

4 Archaeodictyomitra sp.?

3 Crucella sp.

4 Dictyomitra spicularia Aliev

4 Orbiculiforma sp.

5 Praeconocaryomma sp.

Biostratigraphic determination: The Orbiculiforma sp. found in this sample is similar to a form reported by Pessagno from the Albian of California. The Radiolaria is very poorly preserved. It is probably of Albian age.

\section{HOLE 258A}

26-258A-9-2, 114-149 cm.

TA: 4.

RA

3 Globigerinelloides asperus (Ehrenberg)

4 Globotruncana lapparenti (Brotzen)

4 Heterohelix reussi (Cushman)

$4 \quad$ Globigeria sp. (Tertiary forms?)

Biostratigraphic determination: The sample contains Cretaceous and Tertiary forms due to either downhole contamination or reworking. The first possibility is more likely. Whiteinella archaeocretacea Subzone to Marginatruncana concavata Subzone; middle Turonian to early Santonian.

26-258A-9-5, 60-64 cm.

TA: 5 .

\section{RA}

4 Globigerinelloides asperus (Ehrenberg)

3 Globigerinelloides sp.

2 Globotruncana lapparenti (Brotzen)

4 Heterohelix globulosa (Ehrenberg)

4 H. reussi (Cushman)

3 H. rumseyensis Douglas

4 Marginotruncana canaliculata (Reuss)

$3 M$. coronata (Bolli)

Inoceramus prisms abundant.

Biostratigraphic determination: Late Turonian to early Santonian.

\section{SITE 258}

26-258-5-1, 113-115 cm. TA: 5. TA rads: 4 .

\section{RA}

5 Globigerinelloides sp. cf. G. asperus (Ehrenberg)

5 Heterohelix globulosa (Ehrenberg)

$5 \quad H$. reussi (Cushman)

$5 \quad H$. rumseyensis Douglas

1 Marginotruncana coronata (Bolli)

1 M. marginata (Reuss)
3 Whiteinella sp.

2 Archaeochictyomitra squinaboli Pessagno

3 Dictyomitra sp.

Sponge spicules and benthonic forms abundant.

Biostratigraphic determination: Whiteintmla archaeocretacea Subzone to Globotruncana fornicata Subzone; middle Turonian to late Santonian.

\section{6-258-5-3, 75-77 cm.}

TA: 5 .

RA

3 Globigerinelloides asperus (Ehrenberg)

3 Heterohelix globulosa (Ehrenberg)

4 H. reussi (Cushman)

3 H. rumseyensis Douglas

1 Marginotruncana angusticarenata (Gandolfi)

2 M. canaliculata (Reuss)

2 M. coronata (Bolli)

3 M. pseudolinneiana Pessagno

$1 \quad M$. renzi (Gandolfi)

1 Archaeodictyomitra sp.

1 Dictyomitra sp.

Biostratigraphic determination: Late Turonian to Santonian; probably Coniacian.

26-258-6-2, 75-77 cm. TA: 5 . TA rads: 3 .

RA

5 Globigerinelloides asperus (Ehrenberg)

3 Heterohelix reussi (Cushman)

4 Marginotruncana canaliculata (Reuss)

1 Schackoina cenomana (Schacko)

5 Whiteinella sp.

2 Archaeodictyomitra squinaboli Pessagno

3 Dictyomitra sp.

Biostratigraphic determination: Late TuronianSantonian.

26-258-6-4, 60-62 cm.

TA: 5. TA rads: 1 .

RA

1 Archaeoglobigerina blowi Pessagno

3 Archaeoglobigerina sp.

4 Globigerinelloides asperus (Ehrenberg)

4 Globigerinelloides sp.

1 Heterohelix globulosa (Ehrenberg)

3 H. reussi (Cushman)

1 H. rumseyensis Douglas

5 Marginotruncana anguisticarinata (Gandolfi)

4 M. pseudolinneiana Pessagno

3 Whiteinella archaeocretacea Pessagno

4 Whiteinella sp.

1 Bolivonoides $\mathrm{sp}$.

$1 \quad$ Dictyomitra $\mathrm{sp} . ?$

Biostratigraphic determination: Late Turonian to Santonian.

26-258-7-2, 75-77 cm. TA: 5. TA rads: 2 .

RA

4 Archaeoglobigerina sp.

4 Globigerinelloides asperus (Ehrenberg) 
3 Hedbergella sp.

2 Heterohelix globulosa (Ehrenberg)

4 H. reussi (Cushman)

2 Whiteinella archaeocretacea Pessagno

3 Whiteinella sp.

2 Archaeodictyomitra sp.

Inoceramus prisms abundant.

Biostratigraphic determination: Same as above.

26-258-9-1, 70-72 cm. TA: 5 . TA rads: 3 .

RA

4 Globigerinelloides asperus (Ehrenberg)

4 Globigerinelloides sp.

3 Heterohelix globulosa (Ehrenberg)

3 H. reussi (Cushman)

1 H. rumseyensis Douglas

2 Marginotruncana coronata

3 M. pseudolinneiana Pessagno

$4 \quad$ Marginotruncana $\mathrm{sp}$.

1 Schackoina sp.

1 Whiteinella archaeocretacea Pessagno

$4 \quad$ Whiteinella sp.

1 Alievium $\mathrm{sp}$.

1 Archaeodictyomitra squinaboli Pessagno

3 Dictyomitra sp.

2 D. densicostata Pessagno

2 Orbiculiforma quadrata Pessagno

3 Orbiculiforma sp.

Biostratigraphic determination: On the basis of the radiolarian species identified, the age of this sample can be restricted to the late Coniacian or early Santonian.

26-258-10-2, 87-90 cm. TA: 4 . TA rads: 2.

RA

4 Globigerinelloides sp.

2 Heterohelix sp.

$4 \quad$ Whiteinella $\mathrm{sp}$.

2 Archaeodictyomitra squinaboli Pessagno

1 Dictyomitra sp.

Biostratigraphic determination: The sample is very poorly preserved. No specific identification was possible. Via superposition the sample is no older than late Turonian and no younger than early Coniacian.

26-258-11-2,95-97 cm. TA: 4. TA rads: 2.

RA

Globigerinelloides asperus (Ehrenberg)

Globigerinelloides sp.

Heterohelix reussi (Cushman)

$H$. rumseyensis Douglas

Marginotruncana canaliculata (Reuss)

$M$. loeblichae (Douglas)

Whiteinella sp.

Archaeodictyomitra squinaboli Pessagno

Dictyomitra sp.

Biostratigraphic determination: Late Turonian to early Coniacian.
26-258-12-2, $27-29 \mathrm{~cm}$. TA: 1 . TA rads: 3 .

RA

1 Heterohelix sp.

1 Marginotruncana sp.

1 Whiteinella sp.

3 Alievium sp.

2 Archaeodictyomitra squinaboli Pessagno

4 Dictyomitra formosa Squinabol

4 Dictyomitra sp.

3 Orbiculiforma monticelloensis Pessagno

4 Orbiculiforma $\mathrm{sp}$.

3 Pseudoaulophacus sp.

Biostratigraphic determination: Coniacian to early Santonian.

26-258-12-5, 75-77 cm.

TA: 0 .

26-258-13-2, $92-94 \mathrm{~cm}$.

TA: 0 .

26-258-13-4, 98-100 cm.

TA: 0 .

26-258-14-1, $137-139 \mathrm{~cm}$. TA: 4 . TA rads: 3.

RA

2 Hedbergella amabilis Loeblich and Tappan

$3 \quad H$. delrioensis (Plummer)

$5 \quad H$. planispira (Tappan)

3 Hedbergella sp.

Sponge spicules

3 Dictyomitra sp.

3 Orbiculiforma cachensis Pessagno

Biostratigraphic determination: Cenomanian.

26-258-15-2, 79-83 cm. TA: $1 . \quad$ TA rads: 2 .

RA

1 Hedbergella delrioensis (Plummer)

1 Praeglobotruncana delrioensis (Plummer)

Biostratigraphic determination: Rotalipora s.s. Zone; Cenomanian.

26-258-15-5, 35-39 cm. TA: 1 . TA rads: 1 .

RA

1 Hedbergella planispira (Tappan)

1 Praeglobotruncana sp.

Inoceramus prisms abundant.

l Archaeodictyomitra sp.

Biostratigraphic determination: Rotalipora s.s. Zone?; possibly Cenomanian.

26-258-16-2,75-79 cm. TA: 2 . TA rads: 2 .

RA

1 Hedbergella amabilis Loeblich and Tappan

$1 \quad H$. delrioensis (Plummer)

$2 \quad H$. planispira (Tappan)

1 Globigerinelloides bentonensis (Morrow)

1 Globigerinelloides sp. cf. G. caseyi (Bolli, Loeblich and Tappan)

2 Archaeodictyomitra sp. 
Biostratigraphic determination: Rotalipora s.s. Zone; Cenomanian.

\section{6-258-16-5, $75-77 \mathrm{~cm}$.}

TA: 0 .

26-258-17-2, 75-77 cm. TA: 0 . TA rads: 5 .

RA

5 Orbiculiforma maxima Pessagno

5 Orbiculiforma sp.

5 Praeconocaryomma sp.

Biostratigraphic determination: Rotaforma hessi Zone, Cassideus riedeli Subzone; early Cenomanian.

26-258-17-4, 74-76 cm. TA: $0 . \quad$ TA rads: 2 . RA

1 Praeconocaryomma nummulitica Aliev

2 Praeconocaryomma sp.

Biostratigraphic determination: The $P$. nummulitica Aliev is a Berriasian-Valanginian form. It is probably reworked?

26-258-18-2, 75-77 cm. TA: $1 . \quad$ TA rads: 1 . RA

1 Hedbergella planispira (Tappan)

1 Hedbergella sp.

Biostratigraphic determination: Possibly Albian.

26-258-18-4, $71-73 \mathrm{~cm}$.

26-258-20-1, $71-73 \mathrm{~cm}$.

26-258-21-1, 75-77 cm.

26-258-21-3, $75-77 \mathrm{~cm}$.
TA: 0 .

TA: 0 .

TA: 0 .

TA: 0 .
26-258-22-3, 75-78 cm.

TA: 1 .

RA

1 Hedbergella planispira (Tappan)

1 Hedbergella sp.

Biostratigraphic determination: Possibly Albian.

26-258-23-2, 77-79 cm.

TA: 0 .

26-258-24-3, 39-43 cm.

TA: 0 .

26-258-24-5, 47-49 cm.

TA: 0 .

26-258-25-2, 88-90 cm.

TA: 0 .

\section{SUMMARY}

Samples from Site 258, Cores 5 to 11, contain fauna that range in age from late Santonian to middle or early Turonian. Core 12, Section 2, contains Radiolaria that indicate an age no older than Coniacian. Cores 12 and 13 are barren; Core 14, Section 1, contains Cenomanian fauna down to Core 17. Via superposition, it can be established that all strata above Core 12 cannot be older than Coniacian. It is possible that the Turonian is not represented. This view is supported by the fact that Marginotruncana helvetica (Bolli) and $M$. sigali (Reichel), characteristic Turonian species, are absent in the samples.

\section{REFERENCES}

Pessagno, E. A., Jr., 1967. Upper Cretaceous foraminifera from the western Gulf Coastal Plain: Paleont. Am., v. 5 (37), p. 245-445.

Pessagno, E. A., Jr., 1969. Upper Cretaceous stratigraphy of the western Gulf Coast area of Mexico, Texas and Arkansas: Geol. Soc. Am. Mem. 111, p. 1-139.

Pessagno, E. A., Jr., in press. Radiolarian zonation and stratigraphy of the Upper Cretaceous portion of the Great Valley Sequence, California Coast Ranges: Micropaleontology, Spec. Pub. 